\title{
Management of head and neck hemangiomas and venous malformations by bleomycin sclerotherapy
}

\author{
Raluca A. Dragomir ${ }^{1}$, Mihai Liviu Ciofu', Andrada Doscas ${ }^{1}$, Alexandra Carp ${ }^{1}$, \\ Bogdan Dragomir², Nicolae Dobrin ${ }^{3}$, Otilia Boisteanu', Victor-Vlad Costan ${ }^{1}$ \\ ${ }^{1}$ Oral and Maxillofacial Surgery Department, \\ "Grigore T. Popa" University of Medicine and Pharmacy, lasi, Romania \\ ${ }^{2}$ Orthodontics and Facial Orthopedics, \\ "Grigore T. Popa" University of Medicine and Pharmacy, Iasi, Romania \\ 3"Prof. Dr. Nicolae Oblu" Hospital, Neurosurgery, Iasi, Romania
}

\begin{abstract}
Hemangiomas and venous malformations are frequently encountered in the head and neck region, particularly in children. The management of such lesions is especially difficult, considering the characteristics of the age group and the possible complications and sequelae regarding appearance and function. The high esthetic demands of the region, the presence of significant anatomical structures, as well as the concerns for airway maintenance make the choice of treatment extremely challenging. The local injection of a sclerosing agent represents a minimally invasive method that is often used as a first-line treatment of such lesions. The purpose of this manuscript is to review the indications, outcomes and complications of bleomycin sclerotherapy in the treatment of vascular anomalies in the maxillofacial territory.

Keywords: hemangioma, vascular anomalies, low-flow malformation, bleomycin,
\end{abstract} sclerotherapy, head and neck

\section{INTRODUCTION}

Vascular anomalies have the greatest frequency in the head and neck areas and are often encountered in children $(1,2)$. An accurate minimally invasive treatment of this condition is particularly important, considering the predominant age group, the potentially severe complications and debilitating sequelae.

According to the classification of vascular anomalies issued by the International Society for the Study of Vascular Anomalies (ISSVA), two main types of lesions make up this category - vascular tumors (including hemangiomas) and vascular malformations (comprising high-flow and lowflow lesions, as simple or combined malformations) $(1,3-5)$. The treatment options for vascular anomalies consist mainly of medication, sclerotherapy, embolization, laser therapy and surgical removal.
The choice is made according to the age of the patient, the size of the lesion and the expected rate of progression, the anatomical segment involved, the symptoms, as well as the presence or potential for aesthetic and functional impairment or complications $(2,3,6)$.

Sclerotherapy is one of the least invasive treatment methods. It consists of local intralesional injection of a sclerosing agent and it is mainly recommended for hemangiomas, as well as low-flow vascular malformations, of which venous malformations are the most common $(1,7)$. From the multiple known sclerosing agents, bleomycin has been increasingly used in recent studies for the conservative management of head and neck vascular anomalies $(8,9)$.

This manuscript focuses on reviewing the main uses, benefits and complications of bleomycin scle- 
rotherapy for hemangiomas and venous malformations of the maxillofacial territory.

\section{SCLEROTHERAPY}

The local injection of a sclerosing substance inside a hemangioma or venous malformation determines the onset of inflammatory changes and detachment of endothelial cells that eventually determine vessel thrombosis and occlusion, sclerosis and involution of the lesion (9-11). Considering the depth of the lesion, the injections can be performed by imaging guidance, including fluoroscopic or ultrasound examination $(2,3)$. Embolization can be performed before the local injection of sclerosing agent in order to prevent the systemic spread of the substance and to ensure prolonged local action, especially in combined malformations. Multiple sessions are usually necessary in order to achieve good outcomes regarding the reduction in size. Surgery can follow sclerotherapy in order to ensure complete removal of the regressed lesion $(9,11)$.

Multiple sclerosing agents exist that have been used for the treatment of vascular anomalies: absolute ethanol, polidocanol (Aethoxysklerol) foam, sodium tetradecyl sulfate, doxycycline, ethyl-vinyl-alcohol copolymer, cyanoacrylate, 2 poly-hydroxy-ethyl-methacrylate, Ok-432 (Sapylin), bleomycin A2 and bleomycin A5 (Pingyangmycin) $(7,12,13)$. The sclerosing agent of choice is still debated among different authors, since studies showed distinctive outcomes regarding the performance and side effects of the various known substances $(11,13,14)$.

Sclerotherapy is now viewed as the gold standard for the initial treatment of the majority of orofacial venous malformations (13). Although most lesions benefit from this treatment method, the location of the vascular anomaly greatly impacts the indication of sclerotherapy. In this aspect, some authors recommend surgical removal in cases in which the well-known post-injection edema that usually accompanies sclerotherapy may cause complications. This is the case of orbital lesions, when any increase in the orbital volume and pressure may have unwanted permanent effects on vision $(3,15)$. In other instances, surgery may be contraindicated due to the age, the active growing phase unresponsive to medical treatment, increased bleeding risk, delicate anatomical areas that are difficult to access, reconstruct, or are associated with important postoperative functional and aesthetic sequelae (16). In such circumstances, sclerotherapy allows a more conservative approach with minimal complications and good outcomes. Another advantage of sclerotherapy is that it can be used prior to surgery for lesions with unclear borders (7).

\section{BLEOMYCIN}

Bleomycin is classified as an "antitumor antibiotic" with cell-cycle specific and low cytotoxic action. It is commonly used as chemotherapy for head and neck malignant tumors and Hodgkin lymphomas $(7,17,18)$.

The sclerosing effect of bleomycin is attributed to the nonspecific inflammatory reaction induced at the level of the endothelial cells. The first use of bleomycin as a sclerosing agent dates back to 1977 , when Yura et al. administered it for the treatment of cystic lymphangiomas $(13,19)$. Since then, the mechanism, indications, outcomes and side effects of bleomycin sclerotherapy have been better defined. The lifetime total administration of bleomycin should be under $400 \mathrm{mg}$. Its use is contraindicated in patients with a history of renal or pulmonary disorders (20).

\section{INDICATIONS AND OUTCOMES OF BLEOMYCIN SCLEROTHERAPY}

The indications for using bleomycin as a sclerosing agent follow the same criteria as for performing sclerotherapy in general, with the specification that using bleomycin may be more advantageous in certain situations, as compared to other substances associated with more complications $(2,7,20)$. In other words, it is advisable to prefer bleomycin to other agents whenever it is considered critical to avoid certain known complications that may even endanger the patient's life or cause important functional impairment. Bleomycin may be preferred to more aggressive substances, like absolute ethanol, in certain anatomical regions, like the oral cavity, hypopharynx, larynx and parotid gland region. Bleomycin has been used with favorable outcomes for the management of vascular anomalies located in particular regions that are difficult to reconstruct surgically after resection without important aesthetic and functional sequelae, like the eyelid region and the upper airway $(12,18,21)$. Bleomycin sclerotherapy can be performed even in poorly located vascular anomalies, in which surgical or laser access is difficult, including laryngeal lesions (22). An additional benefit for bleomycin sclerotherapy is the possible use in 
growing patients, in which the surgical removal of large lesions, or the use of more aggressive substances for sclerotherapy may cause even more damage due to the presence of scars or associated complications (2). Bleomycin sclerotherapy can also provide an adequate and less invasive management for hemangiomas that are resistant to the initial Propranolol treatment $(16,23)$.

The depth of the vascular anomaly has also been considered in selecting the type of sclerosing agent. One study stated that bleomycin is most efficient in the management of superficial lesions, while the ones in deeper tissues may benefit more from ethanol sclerotherapy (24). Thus, it may be possible that, in order to ensure efficiency, deeper lesions would require a more aggressive treatment using more potent sclerosing agents, even with the increased risk of complications.

Literature reports regarding the response of vascular anomalies to bleomycin sclerotherapy are variable, but a generally positive outcome is presented, with an objective response rate between 65 $100 \%$ according to different studies $(13,18)$. Very good results with few to no complications have been reported by authors using bleomycin A5 (Pingyangmycin) as a sclerosing agent (25). Repeated injections, spaced at one- or two-months intervals, are often required in order to reach the desired outcomes $(13,16,21)$. Liu et al. state that the increased size of the lesion correlated with the need to perform multiple injection sessions (22).

The complete resolution of the lesions is more rarely reported, varying from 20 to $57 \%$ of cases (13). Following the reduction in size of the vascular anomaly, surgical resection can be performed with less morbidity aiming for the complete removal of the lesion, and with the advantage of better outlined borders (7). Laser therapy may also be associated to bleomycin sclerotherapy (8).

\section{SIDE EFFECTS AND COMPLICATIONS OF BLEOMYCIN SCLEROTHERAPY}

The most frequently reported adverse effects associated with bleomycin sclerotherapy are pain at the injection site and soft tissue edema (13). However, their intensity is less than that caused by other sclerosing agents. This is due to the known advantage of bleomycin causing a less prominent and acute inflammatory response compared to other sclerosing substances (8).

Complications following bleomycin sclerotherapy have been reported with different frequencies according to different studies, ranging from 0 to 25 $\%$ (18). The site of the vascular lesion may be in relation to the complication rate, since certain regions in the head and neck may be more prone to developing complications due to their functional involvement and anatomic characteristics (12). In this regard, sclerotherapy of vascular anomalies involving the airway, from the oral cavity to the hypopharynx and larynx, may predispose to airway obstruction due to the variable amount of post-injection edema. The event should be expected, and proper airway protection and surveillance should be initially provided, since swelling of the local tissues is the most common evolution immediately after the injection, regardless of the substance used, although the amount of edema may be more important with certain sclerosing agents $(12,18,22)$.

Less important post-injection swelling has been attributed to bleomycin in comparison with absolute ethanol sclerotherapy (18). As a sclerosing agent, ethanol is a potent substance, but has been linked to numerous complications, including skin and mucosa necrosis, nerve injury, important swelling and even neurological complications if it reaches the systemic circulation $(2,14)$. Therefore, its use may cause important aesthetic and functional impairment in the head and neck region, especially in certain anatomical regions, including the oral cavity, upper airway, parotid region and orbit. Multiple studies underline the advantages of bleomycin over absolute ethanol, due to the absence of debilitating complications (2).

Generally, mild complications have been linked to bleomycin sclerotherapy. Skin infection following administration has been reported by some studies, while others report transitory skin color changes (13).

Other side effects have been attributed to the high systemic presence of the substance. Since bleomycin has a mild cytotoxic effect, it is not generally associated with serious complications like medullary suppression, thrombocytopenia, or a decrease in white blood cells (7). Pulmonary fibrosis is reported in systemic treatment with bleomycin. However, this complication was described by few studies on bleomycin sclerotherapy in cases in which increased cumulated doses were used in children with decreased body surface areas, thus raising concern for treatment adaptation for this category of patients $(7,26)$. One study describes the use of cumulative doses of 100 units $/ \mathrm{m} 2$ without complications (8). Other studies also mention the risk for pulmonary and renal failure, underlining the need for careful monitoring of the functions, especially in children (2). 
The presence of bleomycin in the systemic circulation has been associated with myalgia, malaise, nausea, resembling flu symptoms $(13,18)$. Increased blood levels following local injection can be minimized by performing the embolization of the vascular anomaly in advance to sclerotherapy, especially in lesions with mixed flow characteristics. Imaging guided injections also help prevent inadvertent administration (18). One study recommends that for arterio-venous malformations, bleomycin should be injected interstitially, instead of intravascularly, with the purpose of preventing rapid systemic absorption (8). Additionally, for lowflow vascular anomalies, administration of the substance in the form of foamed bleomycin, obtained with $25 \%$ human serum albumin, may reduce tox-

\section{REFERENCES}

1. Brahmbhatt AN, Skalski KA, Bhatt AA. Vascular lesions of the head and neck: An update on classification and imaging review. Insights Imaging. 2020;11(1):19.

2. Kolokythas A. Vascular Malformations and Their Treatment in the Growing Patient. Oral Maxillofac Surg Clin North Am. 2016; 28(1):91-104

3. Puttgen KB, Pearl M, Tekes A, Mitchell SE. Update on pediatric extracranial vascular anomalies of the head and neck. Childs Nerv Syst. 2010;26(10):1417-33.

4. Enjolras $\mathrm{O}$. Classification and management of the various superficial vascular anomalies: hemangiomas and vascular malformations. J Dermatol 1997;24:701-10.

5. International Society for the Study of Vascular Anomalies (ISSVA). Classification for vascular anomalies. 2014. Available at URL: http:// www.issva.org/classification.

6. Warren TA, Gandhi M, Panizza B. Pictorial review: Vascular anomalies of the head and neck. J Med Imaging Radiat Oncol. 2012; 56(1):8492.

7. Sindel A, Sayan A, Özgür Ö, Sindel T, llankovan V. Percutaneous treatment of orofacial vascular malformations. $\mathrm{Br} J$ Oral Maxillofac Surg. 2018;56(3):206-211.

8. Mack JM, Richter GT, Becton D, Salem O, Hill SEM, Crary SE. Short-term side effects and patient-reported outcomes of bleomycin sclerotherapy in vascular malformations. Pediatr Blood Cancer. 2018; 65(6):e27008.

9. Bothra N, Panda L, Sheth J, Tripathy D. Role of intralesional bleomycin sclerotherapy as the sole or adjunct treatment of superficial ocular adnexal lymphatic malformations. Eye (Lond). 2018;32(1):152-155.

10. Manzano BR, Premoli AM, Santaella NG, Ikuta CRS, Rubira CMF, Santos PSDS. Sclerotherapy as an esthetic indication in oral vascular malformations: a case series. An Bras Dermatol. 2019;94(5):521-526.

11. Stuepp RT, Scotti FM, Melo G, Munhoz EA, Modolo F. Effects of sclerosing agents on head and neck hemangiomas: A systematic review. J Clin Exp Dent. 2019;11(11):e1033-e1044.

12. Seront E, Vikkula M, Boon LM. Venous Malformations of the Head and Neck. Otolaryngol Clin North Am. 2018;51(1):173-184.

13. Horbach SE, Lokhorst MM, Saeed P, de Goüyon Matignon de Pontouraude CM, Rothová A, van der Horst CM. Sclerotherapy for low-flow vascular malformations of the head and neck: A systematic review of sclerosing agents. J Plast Reconstr Aesthet Surg. 2016; 69(3):295-304 icity due to the slow washout and decreased systemic concentration $(18,27)$.

\section{CONCLUSIONS}

As an isolated treatment, or in association to laser or surgical treatment, bleomycin sclerotherapy represents an optimal, minimally invasive method for the management of vascular anomalies in the head and neck. It allows decreasing the overall complications, as well as the functional and aesthetic repercussions associated with initial surgical excision. Among the known sclerotherapy agents, bleomycin has a low complication rate and good response rate, which recommend its use for the majority of lesions with sclerotherapy indication.

Conflict of interest: none declared Financial support: none declared

14. Spence J, Krings T, TerBrugge KG, Agid R. Percutaneous treatment of facial venous malformations: A matched comparison of alcohol and bleomycin sclerotherapy. Head Neck. 2011;33(1):125-30.

15. Tan S, Bialostocki A, Brasch H, Fitzjohn T. Venous malformation of the orbit. J Oral Maxillofac Surg 2004;62(10):1308-1311

16. Chang L, Chen H, Yang X, Jin Y, Ma G, Lin X. Intralesional Bleomycin Injection for Propranolol-Resistant Hemangiomas. J Craniofac Surg. 2018;29(2):e128-e130.

17. National Center for Biotechnology Information. PubChem Database. Bleomycin, $\mathrm{CID}=5460769$, https://pubchem.ncbi.nlm.nih.gov/ compound/Bleomycin (accessed on Mar. 15, 2020).

18. Azene E, Mitchell S, Radvany M, Agrawal N, Eisele D, Weiss C. Foamed bleomycin sclerosis of airway venous malformations: The role of interspecialty collaboration. Laryngoscope. 2016; 126(12):2726-2732.

19. Yura J, Hashimoto T, Tsuruga N, Shibata K. Bleomycin treatment for cystic hygroma in children. Nihon Geka Hokan 1977;46:607e14.

20. Fowell C, Verea Linares C, Jones R, Nishikawa H, Monaghan A. Venous malformations of the head and neck: Current concepts in management. Br J Oral Maxillofac Surg. 2017;55(1):3-9.

21. Shigematsu T, Sorscher M, Dier EC, Berenstein A. Bleomycin sclerotherapy for eyelid venous malformations as an alternative to surgery or laser therapy. J Neurointerv Surg. 2019;11(1):57-61.

22. Liu F, Xiao Y, Wang J. Therapeutic efficacy of intralesional bleomycin injection for laryngopharyngeal haemangioma in adults. Acta Otolaryngol. 2019;139(12):1117-1121.

23. Düzenli Kar Y, Özdemir ZC, Acu B, Bör Ö. Infantile hemangioma: Efficacy of low-dose propranolol and of intralesional bleomycin injection for propranolol non-response. Pediatr Int. 2019; 61(5):459-464.

24. Zhang J, Li HB, Zhou SY et al. Comparison between absolute ethanol and bleomycin for the treatment of venous malformation in children. Exp Ther Med 2013;6:305e9.

25. Luo Q, Zhao F. How to use bleomycin A5 for infantile maxillofacial haemangiomas: clinical evaluation of 82 consecutive cases. J Craniomaxillofac Surg. 2011;39(7):482-6.

26. Park SM, Bae YC, Lee JW, et al. Outcomes of surgical treatment of vascular anomalies on the vermilion. Arch Plast Surg 2016;43:19-25.

27. Helal HA, Mahmoud NA. Effect of foam and liquid bleomycin in the management of venous malformations in head and neck region: $A$ comparative study. J Plast Reconstr Aesthet Surg. 2020;73(1):90-97. 